\title{
Paediatric Surgical Prophylaxis guidelines in the UK. A National survey
}

\section{Sarah Hicks ${ }^{1}$, M Roderick' ${ }^{1}$ A Finn² ${ }^{2}$ S. Vergnanoํ.}

${ }^{1}$ Bristol Children's Hospital, Paediatric infectious diseases and immunology, Bristol, United Kingdom.

${ }^{2}$ University of Bristol, Paediatric infectious diseases and immunology, Bristol, United Kingdom.

Table 1: Surgical prophylaxis guidelines

\section{BACKGROUND}

- Paediatric surgical prophylaxis is often prolonged as shown in several studies ${ }^{1}$

- We conducted a national survey of of surgical antimicrobial prophylaxis guidelines in the UK to establish what is the recommended practice

\section{METHODS}

- 27 surgical units in the UK undertake specialist paediatric surgery

- Antimicrobial prophylaxis guidelines were collected through hospital websites, the MicroGuide app or personal contacts

- Procedures were classified into clean; clean contaminated and contaminated according to the SIGN guidelines.

\section{RESULTS}

Guidelines from 10/24 centres were collected and compared. One centre had no guidelines. Centres with guidelines had separate advice for children with penicillin allergy. The antimicrobial choice was variable: for clean-contaminated procedures , 4/9 centres recommended co-amoxiclav alone or with metronidazole, $3 / 9$ centres recommended cefuroxime and metronidazole, 1/9 centres amoxicillin, metronidazole and gentamicin and 1/9 centres amoxicillin and metronidazole

\begin{tabular}{|c|c|c|c|c|}
\hline $\begin{array}{l}\text { General surgery - } 9 \\
\text { guidelines }\end{array}$ & $\begin{array}{l}\text { No } \\
\text { Prophylaxis }\end{array}$ & $\begin{array}{l}\text { Single } \\
\text { dose }\end{array}$ & Other & SIGN guidelines \\
\hline $\begin{array}{l}\text { Clean procedures-e.g. } \\
\text { splenectomy; Upper GI }\end{array}$ & $5 / 9$ & & & None \\
\hline $\begin{array}{l}\text { Clean contaminated-e.g } \\
\text { interval appendicectomy }\end{array}$ & & $8 / 9$ & 1/9 Induction $+/-24$ hours therapy & Single dose \\
\hline $\begin{array}{l}\text { Complicated } \\
\text { appendicectomy-non } \\
\text { gangrenous }\end{array}$ & & $7 / 9$ & 1/9 Induction $+/-24$ hours therapy & Single dose \\
\hline \multicolumn{5}{|l|}{ Urology - 8 guidelines } \\
\hline $\begin{array}{l}\text { Clean contaminated-e.g } \\
\text { pyeloplasty, VUR surgery }\end{array}$ & & $5 / 9$ & & Single dose \\
\hline Catheter left in situ & & & $\begin{array}{l}4 / 8 \text { induction }+5-7 \text { days oral } \\
\text { prophylaxis or to catheter removal }\end{array}$ & $\begin{array}{l}\text { Single dose }+/ \text { - oral } \\
\text { to catheter removal }\end{array}$ \\
\hline \multicolumn{5}{|l|}{ ENT - 8 guidelines } \\
\hline Tonsillectomy & $2 / 8$ & $\begin{array}{l}1 / 8<2 \\
\text { yrs old }\end{array}$ & $\begin{array}{l}1 / 85 \text { days when suction } \\
\text { diathermy is used }\end{array}$ & None \\
\hline Grommet insertion & & $3 / 8$ & & None \\
\hline \multicolumn{5}{|c|}{ Orthopaedic surgery - 8 guidelines } \\
\hline $\begin{array}{l}\text { Compound fractures or } \\
\text { open surgery of closed } \\
\text { fractures }\end{array}$ & & $2 / 8$ & $\begin{array}{l}\text { Induction }+1-2 \text { doses } \\
1-5 \text { days if metal implants } \\
\text { Induction }+2 \text { doses if implants }\end{array}$ & Single dose \\
\hline
\end{tabular}

\section{CONCLUSIONS}

- The data collection of the remaining surgical guidelines is still on-going

- The majority of paediatric general surgical guidelines suggest single prophylactic antibiotic dose at induction for clean-contaminated and contaminated procedures

- Not all major paediatric surgical units do not have prophylaxis guidelines

- Stewardship programmes need to ensure surgical prophylaxis guidelines are available and followed 